\title{
Index auteurs
}

Vol. 25 - 2009

C C Springer-Verlag France 2010

Abbassi A., 87

Agoumi O., 52

Alvarez Goenaga F., 77

Amrani A., 87

Arssi M., 60, 81

Baron M.P., 105

Ben Hamida F., 17

Benamou P.-H., 1, 5, 32, 42,

74, 114, 116, 117, 142

Benhima M.A., 60, 81, 136

Benjeddi Y., 136

Bonnin M., 133

Bouaziz M., 17

Boutayeb F., 9, 13, 52, 56

Bouyarmane H., 60, 81

Bouysset M., 133

Cooper M.T., 127

Cornu J.-Y., 92

Coudane H., 27

Coughlin M.J., 127

Coury F., 133

Cyteval C., 105

Charvet R., 27

Chbani B., 56

Chojnowski J., 66

Damiano J., 133

Daoudi A., 9, 52
Daum B., 3, 21, 39, 73, 115

De Roover R., 66

Dederichs A., 27

Delagoutte J.-P., 27, 112

Dendane A.M., 87

Desgrippes Y., 40

Douik M., 17

El Andaloussi Y., 81

El Ibrahimi A., 9, 13

Elmrini A., 9, 13, 52, 56

Elleuch M., 44

Elleuch M.H., 44

Etuin P., 66

Fnini S., 81, 136

Fournié B., 119

George T., 27

Ghannouchi M., 17

Ghrib S., 81, 136

Ghroubi S., 44

Goldberg F., 66

Gourinda H., 87

Hassoun J., 60

Jaziri O., 44

Jennart H., 66

Journeau P., 112

Kharrat O., 44
Largab A., 60, 81, 136

Louaste J., 124

Loudyi D., 13

Marzouki A., 13, 56

Meherzi M.H., 17

Messoudi A., 136

Michel B., 27

Nemoz C., 133

Ngamai K., 60

Nouri H., 17

Ouabid A., 66

Ouertatani M., 17

Rachid K., 124

Rodriguez Boronat E., 77

Shimi M., 9, 56

Simon L., 2

Tavernier T., 133

Tebib J.G., 133

Thémar-Noël C., 40

Tonina-Senes A., 105

Viladot Voegli A., 77

Viladot-Péricé R., 77

Yahia A., 44

Zejjari H., 124

Zorman D., 66 